\title{
TEICHMÜLLER SPACES OF GENERALIZED HYPERELLIPTIC MANIFOLDS
}

\author{
FABRIZIO CATANESE - PIETRO CORVAJA
}

\begin{abstract}
In this paper we answer two questions posed by Cat15, thus achieving in particular a description of the connected components of Teichmüller space corresponding to Generalized Hyperelliptic Manifolds $X$. These are the quotients $X=T / G$ of a complex torus $T$ by the free action of a finite group $G$, and they are also the Kähler classifying spaces for a certain class of Euclidean cristallographic groups $\Gamma$, the ones which are torsion free and even.
\end{abstract}

\section{Contents}

Introduction

1. Euclidean cristallographic groups

2. Actions of a finite group on a complex torus $T$

3. Proof of theorem 1

3.1. Concluding remark

References

\section{INTRODUCTION}

The classical hyperelliptic surfaces are the quotients of a complex torus of dimension 2 by a finite group $G$ acting freely, and in such a way that the quotient is not again a complex torus.

These surfaces were classified by Bagnera and de Franchis (BdF08], see also [ES09] and [BPHV] $)$ and they are obtained as quotients $\left(E_{1} \times\right.$ $\left.E_{2}\right) / G$ where $E_{1}, E_{2}$ are two elliptic curves, and $G$ is an abelian group acting on $E_{1}$ by translations, and on $E_{2}$ effectively and in such a way that $E_{2} / G \cong \mathbb{P}^{1}$.

In higher dimension we define the Generalized Hyperelliptic Manifolds (GHM) as quotients $T / G$ of a (compact) complex torus $T$ by a finite group $G$ acting freely, and with the property that $G$ is not a

Date: October 2, 2020.

AMS Classification: 32Q15, 32Q30, 32Q55, 14K99, 14D99, 20H15, $20 \mathrm{~K} 35$.

Key words: Cristallographic groups, group actions on tori, Generalized Hyperelliptic manifolds.

The present work took place in the framework of the ERC Advanced grant $\mathrm{n}$. 340258, 'TADMICAMT'. 
subgroup of the group of translations. Without loss of generality one can then assume that $G$ contains no translations (since the subgroup $G_{T}$ of translations in $G$ would be a normal subgroup, and if we denote $G^{\prime}=G / G_{T}$, then $T / G=T^{\prime} / G^{\prime}$, where $T^{\prime}$ is the torus $\left.T^{\prime}:=T / G_{T}\right)$.

The name Bagnera-de Franchis (BdF) Manifolds was instead reserved in Cat15 and BCF14 for those quotients $X=T / G$ were $G$ contains no translations, and $G$ is a cyclic group of order $m$ (observe that, when $T$ has dimension $n=2$, the two notions coincide, thanks to the classification result of Bagnera-De Franchis in [BdF08]). BdF manifolds of small dimension were studied in Cat15] and Dem16.

Before stating our main theorem, recall first of all that the Teichmüller space $\mathcal{T}(X)$ of a compact complex manifold $X$ is the quotient

$$
\mathcal{T}(X):=\mathcal{C S}(X) / \mathcal{D} \text { iff } f^{0}(X)
$$

of the space of complex structures on the oriented differentiable manifold underlying $X$, which are compatible with the natural orientation of $X$, by the diffeomorphisms of $X$ which are isotopic to the identity.

Recall also that a $K(\Gamma, 1)$ manifold is a manifold $M$ such that its universal covering is contractible, and such that $\pi_{1}(M) \cong \Gamma$.

We have then:

Theorem 1. Given a Generalized Hyperelliptic Manifold $X, X$ is Kähler and its fundamental group $\pi_{1}(X)$ is a torsion free even Euclidean cristallographic group $\Gamma$ (see definitions Q and 13).

Conversely, given such a torsion free even Euclidean cristallographic group $\Gamma$, there are $G H M$ with $\pi_{1}(X) \cong \Gamma$; moreover any compact Kähler manifold $X$ which is a $K(\Gamma, 1)$ is a Generalized Hyperelliptic manifold.

The subspace of the Teichmüller space $\mathcal{T}(X)$ corresponding to Kähler manifolds consists of a finite number of connected components, indexed by the Hodge type of the Hodge decomposition. Each such component is a product of open sets of complex Grassmannians.

\section{EUCLIDEAN CRISTALLOGRAPHIC GROUPS}

Definition 2. (i) We shall say that a group $\Gamma$ is an abstract Euclidean cristallographic group if there exists an exact sequence of groups

$$
\text { (*) } 0 \rightarrow \Lambda \rightarrow \Gamma \rightarrow G \rightarrow 1
$$

such that

(1) $G$ is a finite group

(2) $\Lambda$ is free abelian (we shall denote its rank by $r$ )

(3) Inner conjugation $A d: \Gamma \rightarrow$ Aut $(\Lambda)$ has Kernel exactly $\Lambda$, hence Ad induces an embedding, called Linear part,

$$
L: G \rightarrow G L(\Lambda):=\operatorname{Aut}(\Lambda)
$$

(thus $L(g)(\lambda)=A d(\gamma)(\lambda)=\gamma \lambda \gamma^{-1}, \forall \gamma$ a lift of $g$ ) 
(ii) An affine realization defined over a field $K \supset \mathbb{Z}$ of an abstract Euclidean cristallographic group $\Gamma$ is a homomorphism (necessarily injective)

$$
\rho: \Gamma \rightarrow \operatorname{Aff}\left(\Lambda \otimes_{\mathbb{Z}} K\right)
$$

such that

[1] $\Lambda$ acts by translations on $V_{K}:=\Lambda \otimes_{\mathbb{Z}} K, \rho(\lambda)(v)=v+\lambda$,

[2] for any $\gamma$ a lift of $g \in G$ we have:

$$
V_{K} \ni v \mapsto \rho(\gamma)(v)=A d(\gamma) v+u_{\gamma}=L(g) v+u_{\gamma} \text {, for some } u_{\gamma} \in V_{K} .
$$

(iii) More generally we can say that an affine realization of $\Gamma$ is obtained via a lattice $\Lambda^{\prime} \subset \Lambda \otimes_{\mathbb{Z}} \mathbb{Q}$ if there exists a homomorphism $\rho^{\prime}: \Gamma \rightarrow \operatorname{Aff}\left(\Lambda^{\prime}\right)$ such that $\rho=\rho^{\prime} \otimes_{\mathbb{Z}} K$ (then necessarily $\left.\Lambda \subset \Lambda^{\prime}\right)$.

Remark 3. In the previous formulae in (ii) [2] and in the following we used a shorthand notation, we extend the action of $L(g)$ on $\Lambda$ to $V_{K}$ naturally as $L(g) \otimes_{\mathbb{Z}} I d_{K}$. We shall also often write $g(v):=L(g)(v)$, and $\gamma(v)=\operatorname{Ad}(\gamma)(v)$.

We note that for a cristallographic group $\Gamma$, realizing it via a lattice $\Lambda^{\prime}$ as in (iii) amounts to having all the $u_{\gamma}$ inside the lattice $\Lambda^{\prime}$, in the formula appearing in (ii) [2].

Remark 4. Given a Euclidean cristallographic group $\Gamma$ as above, the exact sequence $(*)$ is unique up to isomorphism, since $\Lambda$ is the unique maximal normal abelian subgroup of $\Gamma$ of finite index.

In fact, if $\Lambda^{\prime}$ has the same property, then their intersection $\Lambda^{0}:=$ $\Lambda \cap \Lambda^{\prime}$ is a normal subgroup of finite index, in particular $\Lambda^{0} \otimes_{\mathbb{Z}} \mathbb{Q}=$ $\Lambda \otimes_{\mathbb{Z}} \mathbb{Q}=V_{\mathbb{Q}}$ and any automorphism of $\Lambda$ which is the identity on $\Lambda^{0}$ is the identity.

Since $\Lambda^{\prime} \subset \operatorname{ker}\left(A d: \Gamma \rightarrow \operatorname{GL}\left(\Lambda^{0}\right)\right), \Lambda^{\prime} \subset \operatorname{ker}(A d: \Gamma \rightarrow \operatorname{GL}(\Lambda))=\Lambda$ : by maximality $\Lambda^{\prime}=\Lambda$.

Remark 5. $L$ makes $\Lambda$ and $V_{K}$ left $G$-modules, and to give an affine realization is equivalent to giving a 1 -cocycle in $Z^{1}\left(\Gamma, V_{K}\right)$ such that $u_{\lambda}=\lambda \forall \lambda \in \Lambda$, since

$$
\begin{gathered}
\rho\left(g_{1} g_{2}\right)=\rho\left(g_{1}\right) \rho\left(g_{2}\right) \Longleftrightarrow L\left(g_{1}\right)\left(L\left(g_{2}\right) v+u_{\gamma_{2}}\right)+u_{\gamma_{1}}=L\left(g_{1} g_{2}\right) v+u_{\gamma_{1} \gamma_{2}} \Longleftrightarrow \\
\Longleftrightarrow u_{\gamma_{1} \gamma_{2}}=u_{\gamma_{1}}+L\left(g_{1}\right)\left(u_{\gamma_{2}}\right)=u_{\gamma_{1}}+g_{1}\left(u_{\gamma_{2}}\right) .
\end{gathered}
$$

(c) Two such cocycles $\left(u_{\gamma}\right),\left(u_{\gamma}^{\prime}\right)$, are cohomologous if and only if there exists a vector $w \in V_{K}$ such that:

$$
u_{\gamma}^{\prime}-u_{\gamma}=\gamma w-w, \forall \gamma \in \Gamma .
$$

(d) Hence two such cocycles are cohomologous if and only if the respective affine realizations are conjugate by a translation in $V_{K}$, since $\rho(\gamma)(v+w)-w=\gamma(v+w)+u_{\gamma}-w=\gamma v+u_{\gamma}+(\gamma w-w)=\gamma v+u_{\gamma}^{\prime}=\rho^{\prime}(\gamma)(v)$. 
Theorem 6. Given an abstract Euclidean cristallographic group there is a unique class of affine realization, for each field $K \supset \mathbb{Z}$.

There is moreover an effectively computable minimal number $d \in \mathbb{N}$ such that the realization is obtained via $\frac{1}{d} \Lambda$.

Proof. Ad $: \Gamma \rightarrow G L(\Lambda)$ makes $\Lambda$ a $\Gamma$-module, a trivial $\Lambda$ module, hence also a $G$-module.

We have seen in remark 5. (b), that an affine realization is given by a cocycle $u_{\gamma}$ in $Z^{1}\left(\Gamma, V_{K}\right)$ such that $u_{\lambda}=\lambda, \forall \lambda \in \Lambda$; and moreover the class of the realization depends only on the cohomology class in $H^{1}\left(\Gamma, V_{K}\right)$.

Consider now the exact sequence of cohomology groups

$$
H^{1}\left(G, V_{K}\right) \rightarrow H^{1}\left(\Gamma, V_{K}\right) \rightarrow H^{1}\left(\Lambda, V_{K}\right)=H o m\left(\Lambda, V_{K}\right) \rightarrow H^{2}\left(G, V_{K}\right) .
$$

Since $G$ is a finite group and $K$ is field of characteristic zero, $H^{1}\left(G, V_{K}\right)=$ $H^{2}\left(G, V_{K}\right)=0$ ([Jac80 pages 355-363): hence we get an isomorphism $H^{1}\left(\Gamma, V_{K}\right) \rightarrow H^{1}\left(\Lambda, V_{K}\right)=H o m\left(\Lambda, V_{K}\right)$.

We look for a cohomology class $\left[u_{\gamma}\right]$ such that its image in $H^{1}\left(\Lambda, V_{K}\right)=$ $\operatorname{Hom}\left(\Lambda, V_{K}\right)$ is the tautological map $\lambda \mapsto \lambda \in V_{K}$, composition of the identity of $\Lambda$ with the inclusion $\Lambda \subset V_{K}$. By the above isomorphism such cohomology class exists, is unique and not equal to zero.

In particular, this applies for $K=\mathbb{Q}$, and since $G$ is finite there is an integer $D$ such that that $u_{\gamma} \in \frac{1}{D} \Lambda$.

Hence we get a cocycle in $H^{1}\left(\Gamma, \frac{1}{D} \Lambda\right)$ whose image in $H^{1}\left(\Gamma,\left(\frac{1}{D} \Lambda\right) / \Lambda\right)$ comes form a unique class in $H^{1}\left(G,\left(\frac{1}{D} \Lambda\right) / \Lambda\right)$. This last class has order dividing $D$, and we let $\delta$ be its order. Hence, setting $D=d \delta$ we obtain that $\left[u_{\gamma}\right]$ comes from $H^{1}\left(\Gamma,\left(\frac{1}{d} \Lambda\right)\right)$.

Remark 7. The above result provides a correct proof for a claim made in [B-C12] and BCF14, answers the question posed in [Cat15], remark 23 , page 312 , and generalizes the result of lemma 18, page 310 ibidem.

We realized later on that the first statement, about the unicity of the affine realization, was proven by Bieberbach in 1912 ([Bieb12]).

We have moreover

Proposition 8. Suppose that $\Gamma$ is an Euclidean cristallographic group, which by theorem [6 is a subgroup of $\operatorname{Af} f\left(V_{\mathbb{Q}}\right)$. Consider a lattice $\Lambda^{\prime} \subset$ $\Lambda \otimes_{\mathbb{Z}} \mathbb{Q}$, such that $\Lambda \subset \Lambda^{\prime}$, and let $\Gamma^{\prime}$ be the corresponding group of affine transformations of $V_{\mathbb{Q}}$, generated by $\Gamma$ and $\Lambda^{\prime}$. Then the group $\Gamma^{\prime}$ sits into a canonical exact sequence

$$
0 \rightarrow \Lambda^{\prime} \rightarrow \Gamma^{\prime} \rightarrow G \rightarrow 1
$$

and such an exact sequence splits if and only if the affine realization of $\Gamma$ is defined over $\Lambda^{\prime}$. 
Proof. The group $\Gamma^{\prime}$ is obtained from $\Gamma$ by adding some translations, which lie in the kernel of $A d: A f f\left(V_{\mathbb{Q}}\right) \rightarrow G L\left(V_{\mathbb{Q}}\right)$, so the image $\operatorname{Ad}\left(\Gamma^{\prime}\right)$ coincides with $G=\operatorname{Ad}(\Gamma)$. Hence we get the exact sequence above.

Assume now that the affine realization is defined over $\Lambda^{\prime}$. Then, for each $g \in G$, let $\gamma \in \Gamma \subset \Gamma^{\prime}$ be any lift of $g$. From $\gamma(v)=L(g) v+u_{\gamma}$ for some $u_{\gamma} \in \Lambda^{\prime}$, we obtain a second lift $\gamma^{\prime} \in \Gamma^{\prime}$ of $g$ by setting $\gamma^{\prime}=u_{\gamma}^{-1} \circ \gamma$, i.e. $\gamma^{\prime}(v)=L(g) v$. Clearly the homomorphism $g \mapsto \gamma^{\prime}$ yields a splitting.

Conversely, if the exact sequence splits, then we have a semidirect product of $\Lambda^{\prime}$ with a group $G^{\prime}$ isomorphic to $G$. Then there is a fixed point $w$ for the action of $G^{\prime}$ on $V_{\mathbb{Q}}$, obtained as $w:=\Sigma_{g \in G^{\prime}} g v$, where $v$ is any vector in $\Lambda$. Choosing $w$ as the origin, we obtain an affine realization of $\Gamma^{\prime}$ defined over $\Lambda^{\prime}$, a fortiori the same holds for $\Gamma$.

Proposition 9. (I) Let $\epsilon \in H^{2}(G, \Lambda)$ be the extension class of $\Gamma$.

Then there is an affine realization of $\Gamma$ defined over $\Lambda^{\prime}$

$\Leftrightarrow \epsilon \otimes_{\mathbb{Z}} \Lambda^{\prime}=0$

$\Leftrightarrow$ there is a fixed point $[w] \in \Lambda^{\prime} / \Lambda$ for the action of $G$ on $V_{\mathbb{Q}} / \Lambda$.

(II) $G$ acts freely on the real torus $T:=\left(\Lambda \otimes_{\mathbb{Z}} \mathbb{R}\right) / \Lambda=V_{\mathbb{R}} / \Lambda$ if and only if $\Gamma$ is torsion free: this means that the subset

$$
\operatorname{Tors}(\Gamma):=\left\{\gamma \mid \exists m \in \mathbb{N}_{+}, \gamma^{m}=1_{\Gamma}\right\}
$$

consists only of the identity.

Proof.

(I) An extension splits if and only if its cohomology class $\epsilon^{\prime} \in$ $H^{2}\left(G, \Lambda^{\prime}\right)=0$ ([Jac80 theorem 6.15, page 365), hence the first assertion follows from proposition 8 ,

The second assertion follows as in the proof of proposition 8 .

(II) if $g$ acts with a fixed point on $T$, there is a lift $\gamma$ of $g$ such that $\gamma$ has a fixed point $w$ in $V$. Then, choosing coordinates such that $w=0$, the action of $\gamma$ is linear, hence the order of $\gamma$ equals the order of $g$.

Conversely, if $\gamma$ has finite order $m$, the vector $w:=\sum_{1}^{m} \gamma^{i}(0)$ is fixed by $\gamma$, hence $[w]$ is fixed by $g$.

Remark 10. Let $X=T / G$ be a Generalized Hyperelliptic Manifold. The action of $g \in G$ is induced by an affine transformation $x \mapsto \alpha x+b$ on the universal cover, hence it does not have a fixed point on $T=V / \Lambda$ if and only if there is no solution of the equation

$$
g(x) \equiv x \quad(\bmod \Lambda)
$$

to be solved in $x \in V$, i.e. to the equation

$$
\lambda \in \Lambda,(\alpha-\mathrm{Id}) x=\lambda-b
$$


in $(x, \lambda) \in V \times \Lambda$. This remark shows that if the action of $G$ on $X$ is free it is necessary that 1 be an eigenvalue of $\alpha=L(g)$ for all non trivial transformations $g \in G$.

\section{ACtions of a finite group on a COMplex torus $T$}

Assume that we have the action of a finite group $G^{\prime}$ on a complex torus $T=V / \Lambda^{\prime}$, where $V$ is a complex vector space, and $\Lambda^{\prime} \otimes_{\mathbb{Z}} \mathbb{R} \cong V$.

Since every holomorphic map between complex tori lifts to a complex affine map of the respective universal covers, we can attach to the group $G^{\prime}$ the group $\Gamma$ of (complex) affine transformations of $V$ which are lifts of transformations of the group $G^{\prime}$.

Proposition 11. $\Gamma$ is an Euclidean cristallographic group, via the exact sequence

$$
0 \rightarrow \Lambda \rightarrow \Gamma \rightarrow G \rightarrow 1
$$

where $\Lambda \supset \Lambda^{\prime}$ is the lattice in $V$ such that $\Lambda:=\operatorname{Ker}(A d), A d: \Gamma \rightarrow$ $G L\left(\Lambda^{\prime}\right)$.

Defining $G^{0}$ to be the subgroup of $G^{\prime}$ consisting of all the translations in $G^{\prime}$, then $G^{0}=\Lambda / \Lambda^{\prime}$, and moreover $G \subset \operatorname{Aut}(V / \Lambda)$ contains no translations.

Proof. $\Lambda$ is a subgroup of the group of translations in $V$, hence it is obviously Abelian, and maps isomorphically onto a lattice of $V$ which contains $\Lambda^{\prime}$. We shall identify this lattice with $\Lambda$.

$\Lambda$ is normal, $G^{0}=\Lambda / \Lambda^{\prime}$ and $G$ embeds in $G L\left(\Lambda^{\prime}\right) \subset G L(\Lambda)$.

Hence the datum of the action of a finite group $G^{\prime}$ on a complex torus $T$ is equivalent to giving:

(1) a cristallographic group $\Gamma$

(2) a $G$ - invariant sublattice $\Lambda^{\prime}$ of the maximal normal abelian subgroup $\Lambda$ of finite index (equivalently, to give a normal such sublattice $\Lambda^{\prime}$ ), so that we may set $G^{\prime}:=\Gamma / \Lambda^{\prime}$

(3) a complex structure $J$ on the real vector space $V_{\mathbb{R}}$ which makes the action of $G$ complex linear.

While the data (1) and (2) are discrete data, the choice of the complex structure $J$ on $V$ may give rise to positive dimensional moduli spaces. We introduce however (see Cat15]) a further discrete invariant, called Hodge type.

Definition 12. (i) Given a faithful representation $G \rightarrow$ Aut $(\Lambda)$, where $\Lambda$ is a free abelian group of even rank $r=2 n$, a $G$ - Hodge decomposition is a $G$-invariant decomposition

$$
\Lambda \otimes \mathbb{C}=H^{1,0} \oplus H^{0,1}, H^{0,1}=\overline{H^{1,0}} .
$$

(ii) Write $\Lambda \otimes \mathbb{C}$ as the sum of isotypical components

$$
\Lambda \otimes \mathbb{C}=\oplus_{\chi \in \operatorname{Irr}(G)} U_{\chi}
$$


Write also $U_{\chi}=W_{\chi} \otimes M_{\chi}$, where $W_{\chi}$ is the irreducible representation corresponding to the character $\chi$, and $M_{\chi}$ is a trivial representation whose dimension is denoted $n_{\chi}$.

Write accordingly $V:=H^{1,0}=\oplus_{\chi \in \operatorname{Irr}(G)} V_{\chi}$, where $V_{\chi}=W_{\chi} \otimes M_{\chi}^{1,0}$.

Then the Hodge type of the decomposition is the datum of the dimensions

$$
\nu(\chi):=\operatorname{dim}_{\mathbb{C}} M_{\chi}^{1,0}
$$

corresponding to the Hodge summands for non real representations (observe in fact that one must have: $\left.\nu(\chi)+\nu(\bar{\chi})=\operatorname{dim}\left(M_{\chi}\right)\right)$.

Definition 13. A cristallographic group $\Gamma$ is said to be even if:

i) $\Lambda$ is a free abelian group of even rank $r=2 n$

ii) considering the associated faithful representation $G \rightarrow \operatorname{Aut}(\Lambda)$, for each real representation $\chi, M_{\chi}$ has even dimension (over $\mathbb{C}$ ).

Remark 14. (i) Given a group action on a complex torus, we obtain an Euclidean cristallographic group which is even, since $\Lambda \otimes_{\mathbb{Z}} \mathbb{R}$ admits a $G$ - invariant complex structure.

(ii) Given an even cristallographic group $\Gamma$, the $G$ - Hodge decompositions of a fixed Hodge type (satisfying the necessary condition $\left.\nu(\chi)+\nu(\bar{\chi})=\operatorname{dim}\left(M_{\chi}\right)\right)$ are parametrized by a (non empty) open set in a product of Grassmannians, as follows.

For a non real irreducible representation $\chi$ one may simply choose $M_{\chi}^{1,0}$ to be a complex subspace of dimension $\nu(\chi)$ of $M_{\chi}$, and for $M_{\chi}=$ $\frac{\chi}{M_{\chi}}$, one simply chooses a complex subspace $M_{\chi}^{1,0}$ of middle dimension.

They must satisfy the open condition that (since $M_{\chi}^{0,1}:=\overline{M_{\chi}^{1,0}}$ )

$$
M_{\chi}=M_{\chi}^{1,0} \oplus M_{\chi}^{0,1} \Longleftrightarrow M_{\chi}=M_{\chi}^{1,0} \oplus \overline{M_{\bar{\chi}}^{1,0}} .
$$

\section{Proof of theorem 1}

1) Let $X=T / G$ be a GHM. Then $X$ is Kähler, since, averaging (by the action of $G$ ) a Kähler metric on $T$ with constant coefficients, we obtain a $G$-invariant one, which descends to the quotient manifold $X$.

2) Since the universal covering of $X$ is the vector space $V$, which is contractible, and $X=V / \pi_{1}(X)$, where $\pi_{1}(X)$ acts freely, we obtain, setting $\pi_{1}(X)=$ : $\Gamma$ that $X$ is a $K(\Gamma, 1)$ manifold.

3) That $\Gamma$ is an Euclidean cristallographic group follows from proposition [11, that $\Gamma$ is torsion free by (II) of proposition 9, and $\Gamma$ is even by (i) of remark 14.

4) If $X^{\prime}$ is also a compact Kähler manifold which is a $K(\Gamma, 1)$, then $X^{\prime}$ admits a Galois unramified covering $T^{\prime}$ with group $G$ such that $T^{\prime}$ is a compact Kähler manifold with the same integral cohomology algebra of a complex torus, hence $T^{\prime}$ is a complex torus, as shown in Cat95, see also Cat15. Hence also $X^{\prime}=T^{\prime} / G$ is a GHM.

5) Moreover, given an Euclidean cristallographic group such that $\Gamma$ is torsion free and even, $V_{\mathbb{R}}$ admits a complex structure by (i) and (ii) 
of remark 14, and the action of $\Gamma$ on $V$ is free since $\Gamma$ is torsion free. Hence for any such complex structure we obtain a quotient $X=V / \Gamma=$ $(V / \Lambda) / G=T / G$ which is a GHM.

6) Observe that the family of GHM is stable by deformation in the large. Indeed, every deformation of a GHM $X=T / G$ yields a deformation of the covering torus $T$ (observe that the covering $T \rightarrow X$ is associated to the unique surjection $\left.\pi_{1}(X) \cong \Gamma \rightarrow G\right)$ and that in AnSt60, Cat02 and Cat04, was proven that a deformation in the large of a complex torus is a complex torus, so that the natural family of $n$-dimensional complex tori is a connected component $\mathcal{T}_{n}$ of the Teichmüller space $\mathcal{T}(T)$ (but not the only one).

7) The connected component $\mathcal{T}_{n}$ of the Teichmüller space of $n$-dimensional complex tori (see Cat02, Cat04 and Cat13 ) is the open set $\mathcal{T}_{n}$ of the complex Grassmann Manifold $\operatorname{Gr}(n, 2 n)$, image of the open set of matrices

$$
\mathcal{F}:=\left\{\Omega \in \operatorname{Mat}(2 n, n ; \mathbb{C}) \mid i^{n} \operatorname{det}(\Omega \bar{\Omega})>0\right\} .
$$

Over $\mathcal{F}$ lies the following tautological family of complex tori: consider a fixed lattice $\Lambda:=\mathbb{Z}^{2 n}$, and associate to each matrix $\Omega$ as above the subspace $V$ of $\mathbb{C}^{2 n} \cong \Lambda \otimes \mathbb{C}$ given as

$$
V:=\Omega \mathbb{C}^{n}
$$

so that $V \in G r(n, 2 n)$ and $\Lambda \otimes \mathbb{C} \cong V \oplus \bar{V}$.

To $V$ we associate then the torus

$$
T_{V}:=V / p_{V}(\Lambda)=(\Lambda \otimes \mathbb{C}) /(\Lambda \oplus \bar{V}),
$$

$p_{V}: V \oplus \bar{V} \rightarrow V$ being the projection onto the first summand.

The crystallographic group $\Gamma$ determines an action of $G \subset S L(2 n, \mathbb{Z})$ on $\mathcal{F}$ and on $\mathcal{T}_{n}$, obtained by multiplying the matrix $\Omega$ with matrices $g \in G$ on the right.

Define then $\mathcal{T}_{n}^{G}$ as the locus of fixed points for the action of $G$. If $V \in \mathcal{T}_{n}^{G}$, then $G$ acts as a group of biholomorphisms of $T_{V}$, and we associate then to such a $V$ the GHM

$$
X_{V}:=T_{V} / G \text {. }
$$

Since the induced family $\mathcal{X} \rightarrow \mathcal{T}_{n}^{G}$ is differentially trivial, we obtain a map $\psi: \mathcal{T}_{n}^{G} \rightarrow \mathcal{T}(X)$.

8) We see that $\mathcal{T}_{n}^{G}$ consists of a finite number of components, indexed by the Hodge type of the Hodge decomposition. Observe in fact that the Hodge type is invariant by deformation, so it distinguishes a finite number of connected components of $\mathcal{T}_{n}^{G}$. That these connected components are just a product of Grassmannians follows from (ii) of remark 14.

9) Assume in greater generality that we have an unramified Galois cover $p: Y \rightarrow X$ such that the associated subgroup $\pi_{1}(Y)=: \Lambda$ is a characteristic subgroup of $\pi_{1}(X)=: \Gamma$, and denote by $G$ the quotient group. Then, via pull back, the space $\mathcal{C S}(X)$ of complex structures $J^{\prime}$ 
on $X$ is contained in the space $\mathcal{C S}(Y)$ of complex structures $J$ on $Y$, and is actually equal to the fixed locus of $G$,

$$
\mathcal{C S}(X)=\mathcal{C S}(Y)^{G}=\left\{J \mid g_{*}(J)=J, \forall g \in G\right\}=\left\{J \mid G \subset \operatorname{Bihol}\left(Y_{J}\right)\right\} .
$$

10) Since $\Lambda$ is a characteristic subgroup, all diffeomorphisms of $X$ lift to $Y$, and we have an exact sequence

$1 \rightarrow G \rightarrow \mathcal{N}_{Y}(G) \rightarrow \mathcal{D}$ iff $(X) \rightarrow 1, \mathcal{N}_{Y}(G):=\{\phi \in \mathcal{D}$ iff $(Y) \mid \phi G=G \phi\}$,

since the diffeomorphisms in the normalizer $\mathcal{N}_{Y}(G)$ of $G$ are the deffeomorphisms which descend to $X$.

11) If $X, Y$ are classifying spaces, then $\operatorname{Diff}(X)^{0}$ is the subgroup acting trivially on $\pi_{1}(X)=\Gamma$, and similarly for $Y$.

12) In our case $G$ acts non-trivially on the first homology, hence we get an inclusion

$$
\mathcal{D} \text { iff }(X)^{0} \subset \mathcal{D} \text { iff }(T)^{0},
$$

as the normalizer subgroup $\mathcal{N}_{Y}(G)^{0}$ of $G$.

13) We consider now Teichmüller space $\mathcal{T}(X)=\mathcal{C S}(X) / \mathcal{D}$ if $f(X)^{0}$. Because of 9) and 12),

$$
\mathcal{T}(X)=\mathcal{C S}(X) / \mathcal{D} \text { iff }(X)^{0}=\mathcal{C S}(Y)^{G} / s N_{T}(G)^{0} .
$$

We get therefore a continuous map $j: \mathcal{T}(X) \rightarrow \mathcal{T}(T)^{G}$, where $\mathcal{T}(Y)^{G}$ is the image of $\mathcal{C S}(Y)^{G}$ inside $\mathcal{T}(Y)$.

14) We want to show that in our case $j$ is a homeomorphism, at least when restricted to the inverse image of $\mathcal{T}_{n}$, which we denote by $\mathcal{T}(X)_{G H}$.

It suffices to observe that $j$ and $\psi$ are inverse to each other, and to show that they are local homeomorphisms. We use remark 14 and proposition 15 of Cat13. In fact, locally for the torus $T$ such that $X=$ $T / G$, Teichmüller space $\mathcal{T}(T)$ is locally the Kuranishi space $\operatorname{Def}(T)$, and in turn $\operatorname{Def}(X)$ is the closed subspace $\operatorname{Def}(X)=\operatorname{Def}(Y)^{G}$ of fixed points for the action of $G$.

Locally there is a surjection $\operatorname{De} f(X) \rightarrow \mathcal{T}(X)$. Composing it with $j$ we get the composition of the inclusion $\operatorname{Def}(X) \subset \operatorname{Def}(T)$ with the local homeomorphism $\operatorname{Def}(T) \cong \mathcal{T}(T)$ : hence the composition is injective.

By 3) remark 14 of Cat13] $\operatorname{Def}(X) \rightarrow \mathcal{T}(X)$ is a local homeomorphism and we can also conclude that $j$ is a homeomorphism with its image $\mathcal{T}(T)^{G}$.

3.1. Concluding remark. We raise the following question: given a generalized Hyperelliptic Manifold $X$, classify the projective manifolds which are a deformation of $X$ (see Dem16] for the special case of Bagnera de Franchis manifolds and [UY76] and [Lan01] for results in dimension 3). 


\section{REFERENCES}

[AnSt60] Andreotti, Aldo; Stoll, Wilhelm. Extension of holomorphic maps. Ann. of Math. (2) 72, 312-349 (1960).

[BdF08] Bagnera, G.; de Franchis, M. Le superficie algebriche le quali ammettono una rappresentazione parametrica mediante funzioni iperellittiche di due argomenti. Mem. di Mat. e di Fis. Soc. It. Sc. (3) 15, 253-343 (1908).

[BPHV] W. Barth, C. Peters, A. Van de Ven, Compact complex surfaces. Ergebnisse der Mathematik und ihrer Grenzgebiete (3), 4. SpringerVerlag, Berlin, (1984); second edition by W. Barth, K. Hulek, C. Peters, A. Van de Ven, Ergebnisse der Mathematik und ihrer Grenzgebiete. 3. Folge. A, 4. Springer-Verlag, Berlin, (2004).

[B-C12] Bauer, Ingrid, Catanese, Fabrizio Inoue type manifolds and Inoue surfaces: a connected component of the moduli space of surfaces with $K^{2}=7, p_{g}=0$. Geometry and arithmetic, 23-56, EMS Ser. Congr. Rep., Eur. Math. Soc., Zürich, (2012).

[BCF14] Bauer, I., Catanese, F., Frapporti, D. Generalized Burniat type surfaces and Bagnera-de Franchis varieties, J. Math. Sci. Univ. Tokyo 22 (2015), 55-111.

[Bieb11] Bieberbach, L. Über die Bewegungsgruppen der euklidischen Räume. (Erste Abhandlung.) Math. Ann. 70, 297-336 (1911).

[Bieb12] Bieberbach, L. Über die Bewegungsgruppen der euklidischen Räume. (Zweite Abhandlung.) Die Gruppen mit einem endlichen Fundamentalbereich. Math. Ann. 72, 400-412 (1912).

[Cat95] Catanese, Fabrizio Compact complex manifolds bimeromorphic to tori. , in 'Abelian varieties' (Egloffstein, 1993), 55-62, de Gruyter, Berlin, (1995).

[Cat02] Catanese, Fabrizio Deformation types of real and complex manifolds. Contemporary trends in algebraic geometry and algebraic topology (Tianjin, 2000), 195-238, Nankai Tracts Math., 5, World Sci. Publ., River Edge, NJ,(2002).

[Cat04] F. Catanese, Deformation in the large of some complex manifolds, I Ann. Mat. Pura Appl. (4) 183, Volume in Memory of Fabio Bardelli, (2004), no. 3, 261-289.

[Cat13] Catanese,Fabrizio, A superficial working guide to deformations and moduli. Handbook of moduli. Vol. I, 161-215, Adv. Lect. Math. (ALM), 24, Int. Press, Somerville, MA, (2013).

[Cat15] Catanese,Fabrizio, Topological methods in moduli theory. Bull. Math. Sci. 5 (2015), no. 3, 287449.

[Dem16] Andreas Demleitner, Classification of Bagnera-de Franchis Varieties in Small Dimensions, arXiv:1604.07678.

[ES09] Enriques, F.; Severi, F.. Mémoire sur les surfaces hyperelliptiques. Acta Math. 32, 283- 392 (1909) and 33, 321-403 (1910).

[Jac80] Jacobson, Nathan Basic algebra. II. W. H. Freeman and Co., San Francisco, Calif., xix+666 pp (1980).

[Lan01] Lange, Herbert Hyperelliptic varieties. Tohoku Math. J. (2) 53 (2001), no. 4, 491-510.

[UY76] Uchida, Koji; Yoshihara, Hisao Discontinuous groups of affine transformations of $\mathbb{C}^{3}$. Tohoku Math. J. (2) 28, no. 1, 89-94 (1976). 
Lehrstuhl Mathematik VIII, Mathematisches Institut der UniverSität Bayreuth, NW II, Universitätsstr. 30, 95447 Bayreuth

E-mail address: fabrizio.catanese@uni-bayreuth.de

Dipartimento di Scienze Matematiche, Informatiche e Fisiche, UniVersitá di Udine, VIA Delle SCIEnze, 206, 33100 Udine

E-mail address: pietro.corvaja@uniud.it 